\title{
PERO YA HABLÉ GALLEGO, LLE DIXEN EU...: ANÁLISIS DE UN CASO DE ALTERNANCIA DE CÓDIGOS EN UNA SITUACIÓN BILINGÜE
}

\author{
NANCY VÁzQuez VeIGA \\ Universidad de A Coruña \\ lxnveiga@udc.es
}

\begin{abstract}
Resumen
There are two main parts in this study about code-switching. The first part focuses on some problems raised by the definition of this language contact phenomenon, such as the identification between language and code, as well as the relationship between code-switching and monolingual norms in bilingual speakers. The second part analyzes an example of Spanish-Galician code-switching, from a socio-discursive perspective. This example, taken from a sociolinguistic interview from the Corpus de lengua hablada de la ciudad de A Coniña, will serve us to revise the traditional distinction between metaphoric and situational code-switching. Additionally, it will serve us to illustrate how code-switching functions as a verbal strategy helping to define and negotiate social relationships, therefore contributing to the fulfillment of conversational cooperation.
\end{abstract}

\section{Introducción}

La investigación sobre la alternancia de códigos $(\mathrm{AC})$ ha experimentado un significativo incremento durante los últimos treinta años. La década de los 70 marca el inicio de un interés generalizado por esta materia. A los primeros estudios, centrados sobre la alternancia español-inglés en los EEUU o sobre la situación de bilingüismo en la India, vinieron a sumarse rápidamente otros, donde se describe una amplia variedad de comunidades bilingües y se estudian pares de lenguas, más o menos próximas desde un punto de vista tipológico, usando distintas aproximaciones teóricas y metodológicas. Nada hacía presagiar que el importante lugar que las interferencias lingüísticas ocupaban en los trabajos de Weinreich (1953) y Haugen (1950), donde apenas se reparaba en la AC, iba en pocos años a ceder su puesto al estudio de la $\mathrm{AC}$, fenómeno de carácter universal, que es en la actualidad uno de los temas más importantes en la investigación sobre el bilingüismo.

El camino que se ha recorrido desde aquellos estudios de la década de los 50 que sugerían que la mezcla o la $\mathrm{AC}$ eran producto de la pereza, la falta de competencia del bilingüe

Quicro manifestar mi agradecimiento a Mauro Fernández Rodríguez por sus atinadas criticas e interesantes comentarios sobre el contenido de este artículo. Además, agradezco a Gabriela Prego Vázquez sus valiosas observaciones y sugerencias en relación con el ejemplo de alternancia de códigos que aquí se comenta. No creo que haga falta mencionar que los defectos que quedan son de mi exclusiva responsabilidad. Este artículo se ha realizado en el marco de los Proyectos PGIDIT02PXIB3051 y BFF2002-04226-C03-01. 
o de su incapacidad para encontrar una determinada palabra en una lengua, ha sido largo ${ }^{2}$. En realidad, las dos corrientes lingüísticas más importantes del siglo XX, el estructuralismo y el generativismo, crearon un ambiente muy poco propicio para el estudio de la variación, de lo heterogéneo. El monolingüismo se presenta como la norma, más que como la excepción en el mundo. Con frecuencia se asume que se trata de un fenómeno universal, hasta el punto de que los individuos bilingües o plurilingües se consideran "bichos raros". Para la tradición estructuralista el que varias gramáticas y diccionarios participen a la vez en el procesamiento del discurso bilingüe ha sido considerado como un peligro, una molestia, una enfermedad (cfr. Muysken, 2000: 1). No obstante, esta orientación básicamente monolingüe no es algo que haya que achacar exclusivamente al desarrollo de las corrientes lingüísticas de mediados del siglo XX, ya que, como sostiene Franceschini (1998: 52), el punto de partida en la mayoría de las teorías lingüísticas continúa siendo el hablante monolingüe, que nunca abandona su lugar de origen y que está rodeado por una mayoría básicamente también monolingüe. Esto resulta en cierto modo inexplicable si tenemos en cuenta que aproximadamente la mitad de la población usa más de una lengua en su vida diaria. Pero nos guste o no, este importante dato no ha tenido todavía consecuencias importantes en lingüística. Está en lo cierto Franceschini (1998: 66) cuando afirma que, si la lingüística se hubiese desarrollado en países de África o del Pacífico, muchos de los cuales tienen cientos de lenguas dentro de sus fronteras, quizá el multilingüismo hubiese tenido un papel más importante en la arquitectura de la teoría lingüística (cfr. Franceschini, 1998: 66).

De todos modos, aunque sepamos hoy mucho más que hace unos años sobre la $\mathrm{AC}$, no podemos afirmar que estemos ante un fenómeno lingüístico de límites claros y perfectamente definidos. No tenemos más que tomar, por ejemplo, las definiciones que Heller (1988a) y Milroy \& Muysken (1995) ofrecen de este fenómeno para observar que bajo la aparente transparencia del término alternancia de códigos y bajo la aparente sencillez de su definición se ocultan algunos problemas que no tienen una fácil solución. Así, según Heller (1988a), estamos ante "el uso de más de una lengua en un único episodio comunicativo"; según Milroy \& Muysken (1995) se trata del "uso alternativo por parte de los bilingües de dos o más lenguas en la misma conversación". Un primer problema surge con el significado de uno de los conceptos que figuran en su denominación, nos referimos al concepto de código: ¿debe tomarse como sinónimo de lengua o de variedad?, ¿se puede identificar código y variedad lingüística?, ¿puede producirse AC, como sugiere Álvarez Cáccamo (1998), sin que alternen las lenguas o las variedades? Un segundo problema tiene que ver con la utilización de la palabra lengua en la definición, por las consabidas dificultades que este término plantea. Finalmente, la presentación de la $\mathrm{AC}$ como una práctica propia del hablante bilingüe también plantea dificultades, debido a la existencia de casos que atestiguan que la AC se puede adquirir directamente, sin necesidad de conocer los estándares de las lenguas o variedades implicadas. Por consiguiente, el concepto de AC no resulta tan fácil de describir como en un principio pudiera parecer; además, como veremos más adelante, no todos los autores lo definen de la misma manera. 
A los problemas relacionados con la descripción de la AC dedicaremos la primera de las dos partes de que se compone este trabajo. En la segunda parte, de carácter esencialmente práctico, comentaremos un fragmento de una entrevista sociolingüística procedente del Corpus de lengua hablada de la ciudad de A Coruña, donde, además de algunas muestras de interferencias lingüísticas del gallego hacia el castellano y del castellano hacia el gallego, encontramos un ejemplo de $\mathrm{AC}$, que será estudiado desde una perspectiva socio-discursiva. El texto seleccionado nos proporciona información sobre las actitudes de los emigrantes gallegos hacia ambas lenguas. El análisis de la $\mathrm{AC}$ nos permitirá revisar la tradicional distinción entre $\mathrm{AC}$ metafórica y situacional, y observar cómo la $\mathrm{AC}$ funciona a modo de estrategia verbal que sirve para definir y negociar las relaciones sociales, contribuyendo así a la consecución de la cooperación conversacional.

\section{Reflexiones en torno a la definición de la alternancia de códigos}

$\mathrm{Al}$ igual que sucede con otros términos, como diglosia, comunidad de habla, no resulta nada fácil superar los escollos que se interponen en la búsqueda de una definición ampliamente aceptada del concepto que nos ocupa, ya que no siempre aparece definido de la misma manera. Así, por ejemplo, Myers-Scotton (2000) llama alternancia de códigos clásica a lo que Muysken (2000) denomina mezcla de códigos. Para Myers-Scotton (2000: 23) la alternancia de códigos clásica es la alternancia entre dos variedades dentro de un mismo constituyente, que realizan los hablantes que tienen suficiente destreza en las dos variedades para producir enunciados monolingües bien formados en cada una de ellas. En cambio, Muysken (2000: 1) usa el término mezcla de códigos para referirse a todos los casos donde en una oración aparecen elementos léxicos y características gramaticales de dos lenguas; reserva el término de $\mathrm{AC}$ para la sucesión rápida de varias lenguas en un simple episodio comunicativo. Por su parte, Álvarez Cáccamo $(1998,2000)$, cuya postura resumiremos más adelante, no considera los casos de mezcla de códigos o, como él dice -siguiendo a Gumperz-, de code-switching style como AC o conmutación de códigos, y utiliza esta última denominación para aludir, entre otros, a aquellos casos en que la alternancia de lenguas es significativa en términos discursivos y pragmáticos, esto es, para designar un fenómeno comunicativo, más que un fenómeno estructural. Por consiguiente, la tradicional inclusión dentro de la etiqueta $\mathrm{AC}$ de los casos de alternancia emblemática o de tipo coletilla, interoracional e intraoracional o mezcla de códigos, recogida en buena parte de los manuales dedicados al estudio del contacto lingüístico, resulta cuando menos bastante discutible. Así pues, si queremos mostrar lo que tienen en común las definiciones de la $\mathrm{AC}$, ni siquiera podemos decir que se trata de un fenómeno característico del discurso bilingüe -piénsese en cómo concibe Álvarez Cáccamo la AC, como fenómeno comunicativo que puede conllevar o no alternancia de lenguas o de variedades (cfr. Álvarez Cáccamo, 1998, 2000). De cualquier forma, la confusión que rodea a su empleo no es algo que deba sorprendernos en exceso, pues uno de los grandes problemas a los que estamos habituados a enfrentarnos los lingüistas es justamente a la falta de una terminología precisa, que se apoye sobre un sistema de conceptos rigurosos.

3 Entre otros, Clyne (2003: 72) se lamenta por la falta de claridad y la polisemia de este término: "The term 'code-switching' has now become so polysemous and unclear that it is necessary to find more precise terms to map out the boundaries and interfaces". 
En general, y de modo similar a lo que pasó con el término diglosia, podemos observar por parte de algunos autores una cierta insatisfacción por el modo en que habitualmente se viene definiendo la $\mathrm{AC}^{3}$. $\mathrm{Al}$ igual que llegó un momento en que prácticamente todas las situaciones de convivencia entre dos o más variedades en el mundo eran calificadas como diglósicas, también en este momento hay una excesiva generalización en el empleo del término $\mathrm{AC}$, sin una detenida reflexión sobre sus orígenes, sobre la conveniencia o no de ver casos de $\mathrm{AC}$ en buena parte de los usos del bilingüe y sobre la imagen que se da del hablante bilingüe y de las lenguas que utiliza. Desde nuestro punto de vista, las críticas más importantes que se han hecho de las definiciones que habitualmente se proporcionan de este término tienen que ver, por una parte, con la excesiva idealización en la presentación tanto de las lenguas o variedades que entran en juego en el discurso con $\mathrm{AC}$, como de las comunidades donde este fenómeno se produce, $y$, por otra, con la capacidad para incorporar dentro de sus fronteras fenómenos que no debería incluir y, sin embargo, excluir otros, que sí debería abarcar. Revisemos algunas de estas críticas.

Para Gardner-Chloros (1995: 68) existe una paradoja que rodea a la AC. Por una lado, el avance en el estudio de la $\mathrm{AC}$ fue consecuencia de la aceptación dentro de la lingüística del bilingüismo como una situación normal, de modo que el comportamiento que se deriva de las situaciones de bilingüismo no podía ser despreciado, tachándolo de arbitrario o aberrante; por otro, se han empleado muchos esfuerzos dentro del campo de la AC para establecer una nueva ortodoxia, que reemplace la antigua de las normas monolingües. Esta nueva ortodoxia consiste en definir la $\mathrm{AC}$ como una forma particular de comportamiento bilingüe especializado, que debe ser distinguida de las manifestaciones "anormales" del bilingüismo, que suponen la influencia de una lengua sobre otra. Según Gardner-Chloros, este nuevo tipo de hablante-oyente ideal, cuya existencia depende de tal discreta alternancia, es un "bicho" tan raro como el hablante monolingüe original de Chomsky. La AC debería ser concebida de manera más comprehensiva, de modo que cubriese un conjunto de fenómenos interlingüísticos, dentro de los cuales la alternancia estricta entre dos sistemas discretos ha de ser la excepción más que la regla (cfr. Gardner-Chloros, 1995: 68). La AC -dice esta autora- se encuentra en una amplia variedad de contextos lingüísticos, que va desde aquellos donde individuos bilingües con formación académica están hablando entre ellos, alternando entre dos códigos que reflejan estrechamente las normas monolingües correspondientes a cada uno de ellos, a las situaciones donde el multilingüismo social es la norma general.

Gardner-Chloros (1995: 86) ve, pues, problemas tanto a la hora de aplicar el término a un tipo de mezcla de lenguas, definido por su independencia gramatical y por el carácter discreto de las variedades que entran en juego, como a la hora de presentarlo como una característica de las comunidades bilingües estables, en contraste con la interpenetración de variedades característica de los procesos de cambio lingüístico. Por un lado, es una utopía considerar la estabilidad de las situaciones bilingües como un absoluto. El único universal que es válido para los lingüistas de todas las escuelas es que las lenguas cambian a través del tiempo y, precisamente, el contacto entre variedades se considera en la actualidad uno de los mecanismos más importantes del cambio. La AC puede ser sólo el primer escalón en el proceso, pero es, sin embargo, parte de él. Y, por otro, la independencia gramatical sobre la que se sostiene la definición de la $\mathrm{AC}$ parece estar en el mejor de los casos en un extremo de un continuo. Los casos, donde los estándares monolingües se apliquen inequívocamente 
a segmentos monolingües dentro del discurso con $\mathrm{AC}$ acabarán siendo identificados por su excepcionalidad. Según Gardner-Chloros (1995: 86),

the idea that there is a one-to-one correspondence between the type of switching which is used, and the degree of bilingual ability of the speaker, must be seen as an oversimplification.

Quizá sea algo arriesgado hablar de "simplificación excesiva", pero lo que sí podemos decir es que esta idea debe ponerse como mínimo en entredicho, pues hay bastantes casos que atestiguan que la competencia en ambas lenguas no parece ser, frente a lo que sugería, por ejemplo, Poplack (1980[1979]), una condición sine qua non para realizar AC o, para ser más precisos, $\mathrm{AC}$ intraoracional o mezcla de códigos ${ }^{4}$. Como muestra de que la alternancia entre dos variedades no lleva necesariamente aparejada una competencia completa en cada una de ellas, sino que también puede ser producida por hablantes que desconocen, por ejemplo, una de las variedades monolingües respectivas, Franceschini (1998) nos relata el caso de una joven de origen germanosuizo, empleada en una tienda de ropa en Zurich. La manera en que esta joven utilizaba la $\mathrm{AC}$ entre italiano y germanosuizo con sus amigos, procedentes de una segunda generación de inmigrantes italianos, no se diferenciaba en nada del modo en que los adolescentes italianos lo hacían. Este testimonio nos lleva a concluir que la $\mathrm{AC}$ puede ser adquirida directamente, sin necesidad de competencia en las lenguas implicadas (cfr. Franceschini, 1998: 57).

El caso del Zaire al que se refieren Meeuwis \& Blommaert (1998) también apoya la tesis de que la $\mathrm{AC}$ puede ser considerada una lengua independiente. Estos autores critican las descripciones de la $\mathrm{AC}$ que parten de la identificación entre lenguas y códigos, y que están supeditadas a un bilingüismo completo por parte los individuos, de ahí que sean partidarios de una visión monolectal de la AC. En el Zaire, la mezcla de códigos constituye por sí misma un código y no existen lenguas subyacentes que tengan alguna realidad interaccional independiente para la mayoría de los hablantes urbanos. Como señalan Meeuwis \& Blommaert (1998: 84), para muchos zaireños que han nacido y crecido en contextos urbanos, la lengua nacional de la región es su lengua madre; pero la lengua que esos niños adquieren es desde el principio una variante marcada por una profusa AC: está repleta de palabras y estructuras francesas, y los niños no la reciben en su variedad "pura". La única forma en que las lenguas nacionales existen es como lenguas con $\mathrm{AC}$. Las pocas ocasiones en que se oye lingala puro o suahelí son en los servicios religiosos, en la radio y en la televisión. Estas variedades son juzgadas por los zaireños como lenguas artificiales, altamente marcadas, y lo que es más, casi ininteligibles. En general, la AC entre las cuatro lenguas, quicongo, lingala, suahelí y

4 Nos parece arriesgado hablar de simplificación porque es difícil establecer principios generales válidos para todos los casos. Los resultados obtenidos en determinados estudios sobre AC no siempre son extrapolables a otros, pues dependen de las características de las comunidades que se estudian y de las variedades que entran en contacto. Así, el que no se pueda considerar la competencia en las dos lenguas o variedades como un requisito necesario para la AC intraoracional no cxcluye la posibilidad de encontrar este tipo de vinculación; por ejemplo, Giacalone Ramat (1995: 62), que ha llevado a cabo algunos estudios en el área italiana, da la razón a Poplack (1980[1979]), cuando afirma que a medida que disminuye la competencia en una lengua aumenta la presencia de la $\mathrm{AC}$ interoracional y se reduce la de la intraoracional. Las generaciones más jóvenes, que poseen una competencia limitada en determinados dialectos, optan por la alternancia de códigos interoracional o por la de tipo etiqueta. 
chiluba (cuatro de las lenguas más importantes del Zaire por su amplia distribución), y el francés es la norma y aparece en todos los niveles, desde en la lengua de los más jóvenes educados en las calles, pasando por la empleada en los contextos más íntimos en el hogar, hasta en la utilizada por los intelectuales y políticos en contextos oficiales.

Este tipo de $\mathrm{AC}$ encaja con dificultad dentro de los límites de la definición de $\mathrm{AC}$ que se da con frecuencia, "uso alternativo de dos o más lenguas", entre otros motivos, porque es difícil presuponer la existencia de códigos y competencias monolingües subyacentes. Este ejemplo también pone en tela de juicio las descripciones de la $\mathrm{AC}$ que parten del supuesto de que se trata de una alternancia "virtuosa" de materiales de dos lenguas distintas y de que esas lenguas distintas son equivalentes a los códigos que se cambian. Como señalan Meeuwis \& Blommaert (1998: 93), conviene tener en cuenta que lo que para los hablantes es el código suele ser bastante diferente de lo que para los lingüistas es su característica más destacada: la diferencia entre lenguas. No se puede confundir la identificación de las lenguas hecha por los lingüistas con la hecha por los hablantes. Debemos tener en cuenta que los códigos mezclados son la norma en algunas comunidades ${ }^{5}$. Lo que para un europeo puede ser calificado como un "buen francés", por su proximidad a la variedad estándar, no coincide necesariamente con lo que para los zaireños es un "buen francés". El francés hablado por los zaireños, que está claramente indigenizado, es para todos los propósitos básicos una lengua zaireña. Como consecuencia de ello, las percepciones sobre lo que es un buen o un mal francés pueden estar basadas sobre criterios locales de competencia y no necesariamente sobre un estándar abstracto. Así, incluso cuando uno habla un francés pobre desde los estándares metropolitanos, su competencia en francés puede ser juzgada por sus iguales como excepcional y como señal de haber recibido una educación y de pertenencia a una élite (cfr. Meeuwis \& Blommaert, 1998: 92). Y es que, como dice Auer (1998: 73-74), sólo para un observador europeo el discurso bilingüe de África posee AC. Las ideologías europeas sobre la lengua nos llevan a buscar los sistemas lingüísticos relativamente homogéneos y codificados que creemos que existen en Europa. Estos sistemas lingüísticos codificados, relativamente homogéneos, puede ser yuxtapuestos en la AC sólo en algunos casos extraordinarios.

Uno de los problemas relacionados con la investigación de la AC es, como apuntábamos anteriormente, la falta de reflexión en torno a sus orígenes. Este tipo de reflexión puede ser importante de cara a aclarar la actual confusión que rodea a sus sentidos. De modo similar a lo que ocurrió con el concepto de diglosia, el significado con el que en la actualidad se suele utilizar tiene poco que ver con el empleo originario del término. Álvarez-Cáccamo (1998, 2000) apunta algunas inexactitudes con respecto a los orígenes del término AC que resulta interesante conocer. Frente a lo que habitualmente se suele pensar, señala que su origen no está en las investigaciones sobre bilingüismo y lenguas en contacto, sino más bien en los estudios sobre comunicación. Además, en los estudios sobre bilingüismo no fue Haugen el primero en utilizar el término $\mathrm{AC}$, como también se piensa a menudo, sino que fue Vogt en un artículo de 1954. El término code-switching probablemente proviene de switching code

5 A este respecto, cfr. Pandharipande (1990), quien describe los usos lingüísticos de una comunidad multilingüe de la India, donde, por ejemplo, el maratí se mezcla con el inglés en contextos vinculados con la modernidad, con el sánscrito en la literatura religiosa y rituales, y con el hindí en reuniones sociales con amigos, para marcar una identidad bilingüe y bicultural. 
que llega a la linguística procedente de los estudios de comunicación. Jakobson adapta la noción de switching code al cambio que un hablante monolingüe o bilingüe debe efectuar, para interpretar (descodificar) el sistema (código) de otra persona.

Álvarez Cáccamo $(1998,2000)$ es partidario de una vuelta a la noción de Jakobson de switching codes, término prestado de la teoría de la información. Este autor critica los trabajos más recientes sobre $\mathrm{AC}$ por igualar códigos con variedades lingüísticas (lenguas, dialectos, etc.), ya que se trata de una ecuación problemática por dos motivos. El primero, es que equiparar la $\mathrm{AC}$ con la alternancia de lenguas supone aceptar que la alternancia de lenguas tal y como la define el lingüista es significativa para los participantes, lo cual anula la posibilidad alternativa de la mezcla de códigos, o como dice Álvarez Cáccamo, de un estilo de $A C$ que ha desarrollado un código propio. De este modo, el alcance de la $\mathrm{AC}$ se ha ampliado hasta incluir la alternancia lingüística no significativa en términos pragmáticos y discursivos (que quedaría excluida en la versión de Jakobson, porque su switching code tiene que ver con la expresión de intenciones y la interpretación de enunciados). El segundo problema es que con las redefiniciones del código de Jakobson orientadas al habla, al igualar códigos con lenguas, también se restringe la extensión del fenómeno. De hecho, las redefiniciones de la situación, diferentes footings o nuevos marcos son frecuentes en todos los contextos sociolingüísticos y no sólo en los bilingües. La noción de código se aproxima mucho a la de señal de contextualización en el sentido de John Gumperz (1982).

Así pues, Álvarez-Cáccamo propone mantener diferenciadas las nociones de código comunicativo y de variedad lingüistica, para recuperar de este modo parte del significado original de códigos como mecanismos de transducción. Mientras que la $\mathrm{AC}$ es un fenómeno comunicativo, la alternancia de variedades o alternancia lingüística $(\mathrm{AL})$ es un fenómeno puramente estructural que puede o no estar ligado a una alternancia o conmutación de códigos. Un código comunicativo es un mecanismo de transducción entre intenciones y enunciados, y entre enunciados e interpretaciones. Son mecanismos generales para poner de manifiesto intenciones en diferentes niveles de organización discursiva. Un código comunicativo dado moviliza y organiza series de indicios de contextualización tanto lingüísticos como no lingüísticos. Una $\mathrm{AC}$ comunicativos es una estrategia detectable de recontextualización por la que se despliegan conjuntos contrastados de señales. En la línea de la teoría de la comunicación, cambiar de código no supone cambiar de lengua o variedad, sino que lleva aparejado la puesta en funcionamiento de otro mecanismo de transducción. Así, una recontextualización de una situación formal a informal puede, por ejemplo, ser activada tanto por un cambio de prosodia como por un cambio de lengua sin excluir otros procedimientos. En definitiva, que la $\mathrm{AC}$ puede o no conllevar alternancia lingüística, del mismo modo que la alternancia lingüística puede no ser interaccionalmente significativa en términos de las intenciones vehiculadas ${ }^{6}$.

Comenzábamos este apartado refiriéndonos a la dificultad de ofrecer una definición ampliamente aceptada de la AC y llegamos al final convencidos de que se trata de una tarea muy compleja. Bastan los ejemplos que hemos revisado y los datos ofrecidos sobre los orígenes del término para ser conscientes de la dificultad de encontrar una descripción de la AC que pueda adaptarse a todos los casos. A medida que se amplían nuestros conocimientos 
sobre la AC más cautelosos debemos ser con los requisitos que hemos de exigir a la hora de utilizar esta etiqueta. Damos la razón a Pfaff (1997: 341) cuando afirma que parece a veces imposible que se pueda realizar cualquier tipo de síntesis sobre la $\mathrm{AC}$, teniendo en cuenta la variación existente en torno a los objetivos de la investigación, a los aspectos que deben ser tratados, a las comunidades de habla y a las variedades lingüísticas que entran en juego en los procesos de $\mathrm{AC}$ :

one may well ask whether code-switching researchers are exploring different parts of the same elephant or different elephants or different species altogether? (ibid.)

Aun así, si queremos disminuir el importante grado de heterogeneidad existente en el ámbito de la $\mathrm{AC}$, consideramos que debe evitarse el empleo de esta etiqueta para aludir a los casos en que las variedades que los hablantes adquieren son códigos mezclados y donde, por consiguiente, deja de tener validez la vinculación que se ha establecido en otras situaciones entre la $\mathrm{AC}$ intraoracional y un alto grado de competencia en cada una de las variedades. Desde nuestro punto de vista, en situaciones como la del Zaire es preferible hablar, como sugieren muchos autores, de mezcla de variedades. No disponemos de tiempo para analizar aquí las implicaciones de la sugerente propuesta de Álvarez Cáccamo de restringir y ampliar simultáneamente la noción de $\mathrm{AC}$, que en última instancia responde a la urgente necesidad de aclarar los sentidos del término. No obstante, hemos de decir que en este caso la batalla contra el peso de la tradición no va a ser sencilla; pensemos que la investigación sobre la AC nació vinculada al discurso bilingüe y no cabe duda de que defender la posibilidad de que, por ejemplo, exista $\mathrm{AC}$ sin alternancia lingüística supone utilizar este concepto con un sentido que no es habitual. Dejaremos en este punto las reflexiones en torno a los problemas que rodean la definición de la $\mathrm{AC}$, para pasar a comentar el fragmento en el que se recoge una muestra de $\mathrm{AC}$ castellano-gallego. La utilización del término $\mathrm{AC}$ para aludir al cambio de variedades que se produce en el texto no creemos que plantee excesivos problemas, en la medida en que este cambio lleva aparejados ciertos efectos comunicativos.

\section{Análisis de una muestra de alternancia de códigos castellano-gallego}

Como dijimos en la introducción, los minutos de conversación que comentaremos proceden de una de las entrevistas sociolingüísticas del Corpus de lengua hablada de la ciudad de $A$ Coruñ $a^{7}$. Centraremos nuestro análisis en dos puntos: en primer lugar, repasaremos brevemente ciertos aspectos estructurales, que tienen que ver con la estructura gramatical de las variedades que se usan, $y$, en segundo lugar, atenderemos al significado social y discursivo de la $\mathrm{AC}$. En el ejemplo que nos disponemos a comentar, mediante la $\mathrm{AC}$, se produce un discurso bilingüe que nos proporciona información sobre la situación social de las lenguas en contacto y sobre el papel que juega cada una de ellas en la interacción entre sus hablantes: 
F. [...] No, pero yo después allá, en Bélgica... Veia un gallego, ;hala! gallego, que hay gente que no, que es ¿no sabes? como es... Ay no, no, no; ellos gallego allá no hablaban. Ay, pero yo si [...] Y yo no, yo me encontraba un gallego... Mira, una vez estaba yo trabajando en el restaurante y llegó un niño de San Pedro de Visma, =

E. $S i$

F. = ¡Qué casualidad (casualidá)! Entonces el niño no sabía que yo estaba en Bélgi$c a,=$

E. $S i$

F. = él sabia que yo estaba en el extranjero, porque era primo de un cuñado mio que murió, entonces yo veo que aquel chiquillo me mira mucho[...] Entonces viene la hermana de mi jefe y dice: "Juli, aquel chico desde que entró te está mirando". Y le salto yo: "Le gustaré" [risa de E]. Pero de esto (d'esto) asi"=

E. $S i$

F. = yo pensando y dice ella: "No, en serio, no, no hables asi que de verdad que en serio, es que ca-, te está comiendo con la vista". Entonces dice ella: "Tú mira con disimulo, a ver si lo conoces y tal". Dije yo: "No mujer". [...]. Y viene, [E sigue riéndose] después voy yo y le dije: "¿Sí?" y dice él: "¿Me conoces?". Y yo: (2) "No”. Y dice él: "Pero, ¿de verdad que no me conoces?". Dije yo: "Pues ahora te me haces una cara conocida, pero aunque me maten, no, no sé de qué te conozco". Y dice: "Son Pepiño". Dije yo: "CCómo vas a ser Pepiño? ¡No me digas...!". 'Claro! Me llevé una alegria... porque (por') ;Claro! dije yo: "Claro, hombre, es que los chiquillos...". Pero ya hablé gallego, lle dixen eu: "Pero, home, é que os nenos cambiades tanto... Tendrias quince anos cando te vin e ahora habia tanto que non te via e tal, jmira os cambios que pegades e tal!" Bueh, ya empecé a hablar todo gallego con él como si nada, ¿y liego? [...] $(\mathrm{M}, 41-59, \mathrm{M})^{8}$

Desde un punto de vista estructural, lo primero que podemos observar en este fragmento es que las dos variedades que entran en juego no son castellano estándar y gallego estándar, sino más bien, castellano con influencias del gallego y gallego con influencias del castellano, o si preferimos, castellano condicionado por el gallego y gallego condicionado por el castellano. Estamos ante lo que García (1976) denomina dialecto agallegado del castellano y dialecto castellanizado del gallego. Se trata de variedades intermedias dentro de un continuo en cuyos extremos estarían el castellano y el gallego estándar; así pues, en este caso tampoco podemos presuponer que la $\mathrm{AC}$ ha de llevar aparejada la existencia de dos variedades perfectamente delimitadas, español estándar y gallego estándar.

En la entrevista dentro de la cual se inscribe este fragmento, la lengua base o la lengua principal de la interacción es el castellano, para ser más precisos, el castellano de Galicia"; se trata de la elección no marcada, mientras que la introducción de fragmentos en gallego es una elección marcada. La entrevistadora, aunque es capaz de entender y de hablar gallego, utiliza normalmente el castellano, dato que conoce la entrevistada, pues, por un lado, el contacto (la persona a través de la cual fue posible concertar la entrevista), una conocida común, también utiliza generalmente sólo el castellano, y, por otro, en alguna ocasión previa a la entrevista, la entrevistada había mantenido con ella una breve conversación en castellano. Además, es la lengua utilizada en el momento en que la entrevistadora llega a la casa

8 La entrevistada pertenece a la clase media y está incluida en el grupo de edad que va de 41 a 59 años.

9 En adelante, utilizaremos simplemente castellano. Debe entenderse que por tal denominación nos estamos refiriendo en realidad al castellano de Galicia. 
donde trabaja la entrevistada, lugar fijado para realizar la entrevista. La charla informal que tiene lugar antes de empezar a grabar es en castellano. Así pues, la entrevista comienza en castellano y finaliza en castellano. Mientras que la entrevistadora procede del medio urbano, en que la lengua empleada es mayoritariamente el castellano, la entrevistada procede del medio rural, donde se habla básicamente el gallego. Sin embargo, hay que tener en cuenta que ésta última emigró hace unos años a Bélgica, donde la lengua utilizada para hablar con su familia, su marido y su hijo, y con otros emigrantes llegados de distintas partes de España era normalmente el castellano, incluso hablaba castellano con algunos conocidos de procedencia gallega. La entrevistada, aunque lleva años viviendo y trabajando en A Coruña como empleada del hogar, en una zona de la ciudad, Juan Flórez, donde vive gente de clase media-alta, por lo general castellano-hablantes, participa frecuentemente en las redes rurales tradicionales, en donde se inserta gran parte de su familia.

Es interesante observar que en el proceso de adaptación de su lengua a la de su interlocutora, la desactivación de la otra lengua, en este caso, el gallego, no se produce totalmente y esto se ve claramente en las interferencias que aparecen en su discurso, interferencias que definiremos, siguiendo a Grosjean (1995: 262), como "desviaciones específicas que realiza una persona en la lengua que está hablando, debido a la influencia de otra lengua «desactivada»" 10 . En este texto las interferencias se producen en todos los niveles y en ambas direcciones, del gallego hacia el castellano y del castellano hacia el gallego. Así, como ejemplos de la influencia del gallego hacia el castellano, cabe mencionar que tanto la entonación como la realización de algunas vocales se corresponden con las del gallego (la - o final de mío se cierra, aproximándose a una -u), también hay que destacar la presencia del llamado pronombre de solidaridad del gallego (te me haces una cara conocida), así como de expresiones tomadas del gallego y adaptadas fonológica y morfológicamente (allá, y luego). En la otra dirección, del castellano al gallego, aunque menos abundantes debido a la corta extensión del fragmento en gallego, podemos hablar de influencia de una lengua sobre otra en los siguientes casos: la anteposición del pronombre personal átono (lle dixen eu por díxenlle eu), el empleo del condicional (tendrias por terias) y la utilización del adverbio (ahora por agora).

Desde una perspectiva gramatical, es más bien poco lo que podemos decir a propósito de la AC. En general, cuando se trabaja con datos procedentes del gallego y del castellano, la analogía tipológica entre las dos lenguas dificulta sobremanera la búsqueda de la lengua base o, en términos de Myers-Scotton, de la lengua matriz, ya que el orden de constituyentes es el mismo y comparten un buen número de palabras funcionales. A pesar de que en este ejemplo no resulta difícil localizar el punto en el que se produce la alternancia, en otros casos, es una tarea realmente complicada por la presencia de palabras ambivalentes en el discurso bilingüe. Por ejemplo, en el siguiente fragmento Hoxe é distinto... sí que o é. Antes comian de todo, de todo. No habia nada que elegir, también procedente de una entrevista del Corpus, tenemos en el medio un enunciado que no podemos identificar como gallego o

10 Como señala Grosjean (1982: 298-299), la mayoría de los investigadores del bilingüismo han propuesto definiciones muy similares de la interferencia. Weinreich (1953) las define como "aquellos ejemplos de desviaciones de las normas de cualquicra de las lenguas, que aparecen en el habla de los bilingües como resultado de su familiaridad con más de una lengua". Haugen (1956) las describe como "el solapamiento de dos lenguas" y Clyne (1972) llama transferencia a la "adopción de cualquier elemento o característica de otra lengua". 
como castellano, ya que puede adscribirse a ambas lenguas: Antes comian de todo, de todo. Este fenómeno se conoce como bivalencia lingüística y consiste, según Woolard (1999), en usar en el discurso bilingüe palabras o enunciados que pueden pertenecer descriptivamente a dos lenguas diferentes. Prego Vázquez (2002: 641) ${ }^{11}$ afirma que

este fenómeno propio de las zonas de contacto lingüístico no ha suscitado tanto interés como la "alternancia lingüística" que ha sido la protagonista estrella de las investigaciones sociolingüísticas cualitativas desde que en los años setenta Gumperz y sus colaboradores se interesaron por su funcionamiento socio-discursivo.

A propósito de las funciones conversacionales que posee la $\mathrm{AC}$ en este texto, lo más destacado es su aparición cuando se introduce en estilo directo parte de un diálogo, que tuvo lugar hace unos años en Bélgica. Con esta estrategia se busca una cierta garantía de "autenticidad" para el discurso ajeno reproducido. Téngase en cuenta que en este episodio se introducen tres voces: la de la entrevistada como locutora, la de la entrevistada como personaje que mantuvo una conversación con Pepiño y la voz de Pepiño. El discurso referido se utiliza aquí para dar la impresión de que se está reproduciendo fielmente la realidad (cfr. Argente Giralt \& Lorenzo Suárez, 1991: 99; Prego Vázquez, 2000: 226). Merece la pena llamar la atención sobre el hecho de que no todos los episodios en estilo directo que se insertan en el discurso reproducen fielmente la variedad en la que tuvo lugar el diálogo. En este caso, observamos que la entrevistada repite un mismo enunciado dos veces, la primera, en castellano, Claro, hombre, es que los chiquillos...., y la segunda, en gallego, Pero, home, é que os nenos (cambiades tanto) Esto muestra, como dice Gumperz (1982: 82), que el habla de los individuos no siempre se reproduce en la lengua que ellos normalmente usan; de ahí que no se puedan establecer reglas del tipo, "El mensaje A se reproduce en el código en el que realmente se produjo", que den cuenta de manera fiable de las funciones conversacionales de ciertos tipos de $\mathrm{AC}$. El establecimiento de reglas de uso que predigan de manera fiable la incidencia de la AC es, según Gumperz (1982: 82), una tarea altamente complicada. A esto también alude Auer (1995: 119) cuando afirma que la lengua de las citas no es necesariamente la lengua utilizada en los enunciados originales, sino que es bastante habitual que la $\mathrm{AC}$ aparezca en casos donde la lengua del fragmento en estilo directo que se reproduce y la lengua realmente utilizada por el hablante sean distintas.

Es interesante mencionar que, en este caso, el paso de una lengua a otra, en concreto, del castellano al gallego, no coincide exactamente con el inicio de la reproducción del diálogo en estilo directo. Esto contradice el punto de vista tradicional según el cual el cambio de lengua está asociado al momento en que se pasa al estilo directo. Obsérvese que la entrevistada dice: Pero ya hablé gallego, lle dixen eu. El lle dixen eu, que, en realidad, traduce la idea contenida en el enunciado anterior emitido en castellano, parece funcionar a modo de preplanificador, en el sentido de que prepara el terreno para la introducción del cambio lingüístico, adelantando el marco del discurso referido. Así pues, en este ejemplo, a los rasgos que anticipan la aparición del estilo directo, voz del narrador o enunciador y verbo de lengua, se suma la alternancia lingüística. La entrevistada cambia de lengua a la hora de introducir una nueva voz (la de la entrevistada como personaje que mantuvo una conver- 
sación con Pepiño). Podemos decir que con la alternancia se abren dos marcos: en primer lugar, se produce un cambio de footing (posicionamiento) (Goffman, 1981), a través del enunciado que prepara el terreno para la reproducción del diálogo en estilo directo, donde se adelanta la elección lingüística del tiempo de la historia, y, en segundo lugar, se produce otro cambio, en el momento en que se pasa a reproducir el estilo directo. La reproducción de este diálogo en gallego es la que provoca una $\mathrm{AC}$ que se anticipa a las palabras desencadenantes; estaríamos ante lo que Clyne $(1967,1972)$ denomina el accionador (triggering) de la alternancia.

De cara a comentar los usos situacionales o metafóricos de la $\mathrm{AC}$, nos parece fundamental detenernos en la distinción entre tiempo de la historia -término que acabamos de introducir hace un momento- y tiempo del relato, pues la AC tiene, desde nuestro punto de vista, una función diferente en cada momento. En el tiempo de la historia, la alternancia que se produce nos recuerda a uno de los tipos de AC propuesta por Gumperz y sus colaboradores, en los trabajos ya clásicos -Blom \& Gumperz (1972) y Gumperz \& Hernández Chávez (1975)-, esto es, una alternancia situacional, donde el cambio lingüístico está relacionado con factores contextuales, como los participantes, el tema, etc. Alguno de los componentes del evento de habla -escenario, temas, participantes- actúa como factor activador de este tipo de alternancia. Anteriormente la entrevistada había contado que, en Bélgica, trabajaba en un restaurante de emigrantes españoles de Burgos, así que la lengua que normalmente empleaba con sus jefes era el castellano y la que utilizaba con los clientes, el francés, excepto en el caso de que fuesen de procedencia española. Así pues, el cambio al gallego se produce cuando entra en escena un nuevo participante, aunque no de manera inmediata, porque durante los primeros minutos se produce una interesante negociación de la lengua en la que tendrá lugar el resto de la interacción. Si nos atenemos a lo que cuenta la entrevistada, la lengua seleccionada para abrir la interacción es el castellano; el paso del they code al we code no se produce hasta el momento en que su interlocutor se identifica, para lo cual proporciona dos pistas, revelar su nombre y cambiar de lengua: Son Pepiño A partir se ese momento se produce una identificación del emisor y del receptor como miembros de un determinado grupo. En la elección de variedades hay implicada una distinción simbólica entre el nosotros y el ellos. En este caso, la lengua minoritaria, el gallego, se identifica con el nosotros (we code) y se asocia, como dice Gumperz (1982: 66), con actividades informales, intragrupales, personalizadas; en cambio, la lengua mayoritaria, el castellano, sería la variedad de ellos (they code), y se asocia con las relaciones exteriores al grupo, de carácter más formal y menos personal. En cierto sentido recuerda a la distribución de usos lingüísticos de Paraguay que describe Rubin -recordemos que la situación lingüística de Paraguay fue descrita por Fishman como un ejemplo de diglosia-:

O español é a lingua das ocasións formais; emprégase sempre para os asuntos do goberno, na conversa con descoñecidos ben vestidos, con estranxeiros e na meirande parte das transaccións comerciais. Usase o guarani, en cambio, cos amigos, criados e descoñecidos non demasiado ben vestidos, no confesionario, para o humor e para o amor, e na meirande parte das ocasións ordinarias. (Apud Wardhaugh 1992[1986]: 122. La cursiva es nuestra).

Se trata, pues, de un cambio de código producido por el interlocutor y por el tipo de relación que se establece entre él y el otro participante. Aunque esta alternancia la hemos descrito como situacional, sin embargo, su dimensión afectiva nos hace pensar en la alter- 
nancia metafórica, como sabemos, el otro tipo de alternancia que distingue Gumperz y sus colaboradores. Se cambia de código porque se produce una redefinición de la situación: de formal a informal. Por otro lado, si nos fijamos en la función conversacional que dicha alternancia lleva aparejada en el tiempo del relato, en el momento en que tiene lugar la entrevista, nos encontraríamos con una alternancia metafórica, cuya función parece ser la de enriquecer las posibilidades expresivas del discurso, pues el contexto de interacción no se modifica. De hecho, el cambio lingüístico producido en el discurso referido es un ejemplo típico de AC metafórica, así como también lo son los que se asocian a efectos irónicos o de énfasis. Tanto en el tiempo del relato como en el tiempo de la historia, la entrevistada y la entrevistadora seleccionan la lengua que mejor reproduce su intencionalidad comunicativa. Así, por ejemplo, la entrevistada echa mano de la alternancia para representar sus identidades castellana y gallega simultáneamente, o si preferimos, sus identidades rural y urbana (cfr. Prego Vázquez, 2000: 187). Como vemos, el establecimiento de una distinción rígida entre $\mathrm{AC}$ situacional y metafórica es difícil de mantener, pues, aunque es cierto que la entrada en escena de un nuevo participante provoca que se produzca un cambio de lengua, éste no se produce hasta que uno de ellos se identifica y es a partir de ese momento cuando el cambio lleva asociados determinados significados, como la dicotomía nosotros (we) / ellos (they), vinculados con frecuencia a la alternancia metafórica. Damos, pues, la razón a Auer (1984: 90), cuando dice que no hay razón para trazar una línea divisoria entre las dos categorías de AC; entre ellas existe más bien una diferencia de grado. La distinción entre AC situacional y metafórica debe ser criticada, según Auer (1984: 91), desde ambos extremos: en el extremo situacional, la relación entre elección lingüística y características situacionales es menos rígida, más abierta a renegociación; en el extremo metafórico, las cosas son menos individualistas, menos independientes de la situación. Esta distinción debería ser remplazada por un continuo, de lo contrario, la mayor parte de las veces su aplicación será problemática.

En general, el comportamiento de esta entrevistada podría ser explicado a la luz de la teoría de acomodación del habla formulada por Giles y sus colaboradores, basada en la noción de convergencia y divergencia entre los hablantes (Giles \& St. Clair, 1979). Normalmente, cuando los hablantes desean la aprobación de su interlocutor, tienden a adaptar su habla, con el objeto de estrechar la distancia social entre ellos. Esta adaptación se consigue modificando un amplio conjunto de características lingüísticas entre las que se encuentra la AC. Como dice Giacalone Ramat (1995: 49-50), el hecho de que la teoría pueda aplicarse a las interacciones monolingües es un argumento a favor de su validez, incluso si no es posible explicar todas las elecciones en términos de acomodación o no al destinatario. Como decíamos antes, el paso del gallego al castellano se produce cuando su interlocutor se identifica como un individuo de su pueblo (Son Pepiño), de tal modo que, en ese momento, el castellano pasa a ser la elección marcada, mientras que el gallego sería la elección no marcada. Estaríamos ante lo que Myers-Scotton (1988: 160) denomina elecciones secuenciales no marcadas pues se pasa de una elección no marcada (el castellano) a otra también no marcada (el gallego). La nueva información acerca de una determinada identidad étnica hace que se produzca una redefinición del intercambio (Myers-Scotton, 1988: 161). Eso en el tiempo de la historia; en el tiempo del relato ocurriría lo contrario: la elección no marcada sería el castellano. De ahí que la alternancia que se produce en el tiempo del relato (lle dixen eu), previa a la introducción del estilo directo, constituya una elección marcada. Como señala 
Myers-Scotton (1988: 170), ésta es una de las muchas variantes de la alternancia como elección marcada, que habitualmente se caracterizan por su brevedad. En este caso vemos que la alternancia puede estar motivada por uno de los factores a los que alude Myers-Scotton (1988: 170), esto es, por el deseo de que el interlocutor conozca que el que habla tiene una personalidad multifacética: "No sólo yo soy X, sino que también soy Y". Esta idea vendría reforzada por el hecho de que la entrevistada no se conforma con mostrar en el tiempo de la historia sus habilidades para emplear otra lengua, sino que lo anticipa al tiempo del relato. El carácter enfático del enunciado, que, como expusimos, posee un significado referencial muy próximo al que lo precede en castellano, parece estar en consonancia con sus deseos de subrayar esa identidad mixta. Esta hablante parece sentirse orgullosa de su competencia lingüística alternante, no compartida con otros gallegos que también emigraron a Bélgica. Su deseo de mostrar una competencia lingüística en ambas lenguas es lo que probablemente la lleva por un momento a transgredir las reglas que rigen su elección lingüística.

En definitiva que, salvo este cambio momentáneo en el tiempo del relato que acabamos de comentar, lo que parece motivar el cambio de lenguas es la búsqueda de un acercamiento a sus interlocutores del tiempo de la historia (Pepiño) y del relato (la entrevistadora), con objeto de establecer una relación más próxima con él o ella y facilitar la comunicación en un nivel interpersonal. La presencia de un comportamiento lingüístico divergente, donde cada participante usase un código, podría ser tomado como una señal de menor cooperación conversacional (cfr. Giacalone Ramat, 1995: 50). Generalmente hablando, los hablantes bilingües tienen a acomodarse más que a construir barreras (vid. Heller 1988a). Aquí tenemos un ejemplo en el que la AC funciona, como señala Heller (1998b), como una estrategia para definir y negociar las relaciones sociales, convirtiéndose en un elemento crucial para el mantenimiento, creación o disolución de las fronteras sociales. Puede funcionar a modo de estrategia verbal para establecer cooperación conversacional, como en este caso, o para prevenir su establecimiento.

La reflexión metadiscursiva que la entrevistada realiza nos proporciona información sobre los usos lingüísticos de la mayor parte de estos emigrantes: ellos gallego allá no hablaban. Esta práctica metadiscursiva se produce como consecuencia de la negativa de algunos emigrantes gallegos a hablar su lengua. Minutos antes se había referido al fuerte rechazo que su marido experimentaba hacia el gallego: ni siquiera estaba dispuesto a hablarlo en el ámbito doméstico y le disgustaba que su mujer lo utilizase delante de su hijo. En el caso del marido, así como de otros emigrantes, que abandonaron sus hogares por motivos de carácter laboral, se produce una identificación entre el poder económico y la lengua o las lenguas de prestigio (el castellano y el francés). Como dice Freixo Mariño (1997: 563-564):

A consideración do galego como sinónimo de pobreza, paro e emigración que eles mesmos tiveron que sufrir, fai que non sexa xulgada no seu xusto termo, é dicir, como un trazo de identidade da cultura e a historia dun pobo, como algo que é preciso manter e fomentar entre as novas xeracións e do que habería que sentirse fachendoso.

\section{Consideraciones finales}

A través de la información contenida en este fragmento sobre el empleo de las lenguas, podemos observar que los bilingües en su vida diaria se encuentran, como afirma Grosjean (1995: 262), en varios puntos de un continuo situacional que los lleva a utilizar sus lenguas 
de una manera determinada. En un extremo de este continuo, los hablantes bilingües se sitủan en lo que Grosjean (1995: 261) denomina un modo de lengua totalmente monolingüe, pues se están dirigiendo a hablantes monolingües tanto de la lengua $\mathrm{A}$ como de la lengua $\mathrm{B}$, de ahí que deban ceñirse a una sola lengua ( $\mathrm{A}$ o B). En el otro extremo del continuo, se sitúan en un modo de lengua bilingüe, ya que están interactuando con bilingües con los que comparten sus dos lenguas (A y B) y con quienes normalmente alternan o mezclan sus lenguas. Nuestra entrevistada se encontraría en el modo bilingüe, porque tanto su interlocutor en el tiempo de la historia como su interlocutora en el tiempo del relato conocen perfectamente las dos variedades que ella maneja. Los bilingües difieren en la manera en que "viajan" a lo largo del continuo; algunos raramente se encuentran en la zona bilingüe, mientras que otros excepcionalmente dejan esa zona (cfr. Grosjean, 1995: 262). El comportamiento lingüístico de la entrevistada y de Pepiño, a diferencia del de otros emigrantes gallegos en Bélgica, muestra su resistencia a mantenerse en la zona monolingüe y sus deseos de reactivar los lazos de cohesión grupal.

A lo largo de este trabajo hemos visto que en estos momentos, en que el estudio de la $\mathrm{AC}$ ha llegado a su madurez, los investigadores reclaman una mayor precisión a la hora de utilizar esta denominación. Se ha pasado de pensar que la AC era una característica del discurso bilingüe derivada de una escasa competencia en una de las variedades, de la pereza de los hablantes bilingües, etc., a pensar que se trata de un fenómeno perfectamente estructurado, sólo al alcance de bilingües equilibrados. Si bien las primeras ideas sobre la $\mathrm{AC}$ han de ser desterradas, las segundas, deben ser como mínimo revisadas. Hemos mostrado que determinados rasgos que hace pocos años se consideraban básicos para definir la $\mathrm{AC}$ no son, en la actualidad, considerados de igual manera por parte de algunos investigadores. Así, al menos dos de las características de la AC esbozadas por Argente Giralt \& Lorenzo Suárez (1991: 92), "que cada variedad posee plena autonomía" y "un certo grado de acceso ás variedades implicadas por parte dos falantes" no serían válidas para describir ciertas manifestaciones del fenómeno que aquí hemos revisado. Otra cuestión es si en esos casos deberíamos seguir utilizando la etiqueta AC.

Para finalizar nos gustaría apuntar que en España, en los próximos años, encontraremos un banco de datos importantísimo para el estudio en general de situaciones de contacto lingüístico, debido al crecimiento del número de inmigrantes que llegan a las distintas ciudades de nuestro territorio. Es importante, pues, que fundamentalmente los que ejerzan su profesión en el ámbito de la enseñanza aprendan a respetar la diversidad y a considerar los fenómenos de contacto lingüístico como una consecuencia natural de la convivencia entre dos o más lenguas. Fernández Rodríguez (1997: 87) hace hincapié en

la necesidad de una mayor tolerancia, especialmente por parte de los profesores, hacia las variedades desarrolladas en situaciones de contacto. Estas variedades no suponen ningún tipo de degeneración de la lengua, como frecuentemente se afirma.

De ahí que resulte fundamental difundir los conocimientos existentes sobre este tema. Es mucho lo que lo especialistas saben sobre la $\mathrm{AC}$ y son muchos los mitos que han sido desterrados, pero también es importante que al menos parte de sus conocimientos lleguen al resto de la sociedad, ya que acabar con la consideración de este fenómeno como algo anormal y extraordinario, y corregir la sobrevaloración que tradicionalmente se viene ha- 
ciendo del dominio de las variedades estándares de las lenguas de un bilingüe, no es algo que se consiga fácilmente.

\section{Apéndice. Convenciones de transcripción}

$\mathrm{E}$
$\mathrm{H}$
$\ldots$
()

entrevistador/a hablante (entrevistado/a) indican pausa o vacilación

() forma pronunciada (se escribe la forma estándar y entre paréntesis la realmente pronunciada)

(número) recoge la duración de la pausa numéricamente en segundos

[ ] comentario no lingüístico o paralingüístico

$=\quad$ sucesión inmediata entre dos emisiones de un hablante

bla bl- palabra truncada

\section{Referencias bibliográficas}

Álvarez Cáccamo, Celso (1998): "From 'Switching Code' to 'Codeswitching': Towards a Reconceptualisation of Communicatives Codes". En Peter Auer (ed.), págs. 29-48.

Álvarez Cáccamo, Celso (2000): "Para um modelo do "code-switching" e a alternância de variedades como fenómenos distintos: dados do discurso galego-português / espanhol na Galiza", Estudios de Sociolingüística, 1(1), págs. 111-128.

Argente Giralt, Joan A. y Anxo M. Lorenzo Suárez (1991): "A relevancia social da alternancia lingüística", Cadernos de lingua, 3, págs. 91-109.

Auer, Peter (1984): "On the Meaning of Conversational Code-Switching". En Peter Auer \& Aldo di Luzio (eds.), Interpretative Sociolinguistics, 87-112. Tübingen: Narr.

Auer, Peter (1995): "The Pragmatics of Code-Switching: A Sequential Approach". En Lesley Milroy \& Pieter Muysken (eds.), págs. 115-135.

Auer, Peter (1998): “Introduction: Bilingual Concersation Revisited". En Peter Auer (ed.), págs. 1-24. Auer, Peter (ed.) (1998): Code-Switching in Conversation. Language, Interaction and Identity. Londres / Nueva York, Routledge.

Benson, Erica J. (2001): "The Neglected Early History of Codeswitching Research in the United States", Language \& Communication, 21, págs. 23-36.

Blom, Jan Petter y John Gumperz (1972): "Social Meaning in Linguistic Structures: Code-Switching in Norway". En John Gumperz \& D. Hymes (eds.), Directions in Sociolinguitics, págs. 407-434. Nueva York, Holt, Rinehart \& Winston.

Clyne, Michael (1967): Transference and Triggering. La Haya, Nijhoff.

Clyne, Michael (1972): Perspectives on Language Contact: Based on a Study of German in Australia. Melburne, Hawthorne Press.

Clyne, Michael (2003): Dynamics of Language Contact. Cambridge, Cambridge University Press.

Fernández Rodríguez, Mauro (1997): "Algunas perspectivas sociolingüísticas sobre la emigración". En Carlos Hernández Sacristán y Ricard Morant Marco (eds.), Lenguaje y Emigración, págs. 7197. Valencia: Universidad de Valencia.

Franceschini, Rita (1998): "Code-Switching and the Notion of Code in Linguistics". En Peter Auer (ed.), págs. 51-72. 
Freixo Mariño, María Xosé (1997): "Lingua e prestixio social en comunidades emigrantes". En Benigno Fernández Salgado (ed.), Actas do IV Congreso Internacional de Estudios Galegos, vol. I, Lingua, págs. 557-566. Oxford, Centre for Galician Studies.

García, Constantino (1976): "Interferencias lingüísticas entre gallego y castellano", Revista de la Sociedad Española de Lingüística, 6/2, págs. 327-343.

Gardner-Chloros, Penelope (1995): "Code-Switching in Community, Regional and National Repertoires: The Myth of the Discreteness of Linguistic Systems". En Lesley Milroy \& Pieter Muysken (eds.), págs. 68-89.

Giacalone Ramat, Anna (1995): "Code-Switching in the Context of Dialect/Standard Language Relations". En Lesley Milroy \& Pieter Muysken (eds.), págs. 45-67.

Giles, Howard y R. St. Clair (1979): Language and Social Psychology. Oxford, Blackwell.

Goffman, Erving (1981): Forms of Talk. Oxford, Blackwell.

Grosjean, François (1982): Life with Two Languages: An Introduction to Bilingualism. Cambridge, Harvard University Press.

Grosjean, François (1995): "A Psycholinguistic Approach to Code-Switching: The Recognition of Guest Words by Bilinguals". En Lesley Milroy \& Pieter Muysken (eds.), págs. 259-275.

Gumperz, John J. (1982): Discourse Strategies. Cambridge, Cambridge University Press.

Gumperz, John y E. Hernández Chávez (1975): "Cognitive Aspects of Bilingual Communication". En

Hernández Chávez et alii (eds.), El lenguaje de los Chicanos, págs. 111-125. Arlington, Center for Applied Linguistics.

Haugen, Einar (1950): "The Analysis of Linguistic Borrowing", Language, 26(21), págs. 210-231.

Haugen, Einar (1956): Bilingualism in the Americas: A Bibliography and Research Guide. Alabama,

University of Alabama Press.

Heller, Monica (1988a): "Introduction". En Monica Heller (ed.), págs. I-24. i

Heller, Monica (1988b): "Strategic Ambiguity: Code-Switching in the Management of Conflict". En

Monica Heller (ed.), págs. 77-96.

Heller, Monica (ed.) (1988): Codeswitching. Anthropological and Sociolinguistic Perspectives, Berlín

/ Nueva York / Amsterdam, Mouton de Gruyter.

Meeuwis, Michael y Jan Blommaert (1998): “A Monolectal View of Code-Switching. Layered Code-

Switching among Zairians in Belgium”. En Peter Auer (ed.), págs. 76-98.

Milroy, Lesley y Pieter Muysken (1995): "Introduction: Code-Switching and Bilingualism Research".

En Lesley Milroy y Pieter Muysken (eds.), págs. 1-14.

Milroy, Lesley y Pieter Muysken (eds.) (1995): One Speaker, Two Languages. Cross-Disciplinary

Perspectives on Code-Switching Cambridge, Cambridge University Press.

Muysken, Pieter (2000): Bilingual Speech. A Typology of Code-Mixing. Cambridge / Nueva York, Cambridge University Press.

Myers-Scotton, Carol (1988): "Codes Switching as Indexical of Social Negotiations". En Monica

Heller (ed.), págs. 151-186.

Myers-Scotton, Carol (2000): "The Matrix Language Frame Model: Developments and Responses".

En Rodolfo Jacobson (ed.), Codeswitching Worldwide II, págs. 23-58. Berlín /Nueva York,

Mouton de Gruyter.

Pandharipande, Rajeshwari (1990): "Formal and Functional Constraints on Code-Mixing". En Rodolfo Jacobson (ed.), Codeswitching as a Worldwide Phenomenon, págs. 15-31. Nueva York, Peter

Lang.

Pfaff, Carol W. (1997): "Contacts and Conflicts: Perspectives from Code-Switching Research". En Martin Pütz (ed.), Language Choices. Conditions, Constraints, and Consequences, págs. 341-360. Amsterdam, John Benjamins.

Poplack, Shana (1980[1979]): "Sometimes I'll Start a Sentence in Spanish y TERMINO EN ESPAÑOL: Toward a Typology of Code-Switching", Linguistics, 22, págs. 99-136. 
Prego Vázquez, Gabriela (2000): Prácticas discursivas, redes sociales e identidades en Bergantiños (Galicia): la interacción comunicativa en una situación de cambio sociolingüistico. Tesis doctoral inédita, Departamento de Galego-Portugués, Francés e Lingüística. A Coruña, Universidad de A Coruña.

Prego Vázquez, Gabriela (2002): "La bivalencia lingüística gallego/español como estrategia discursiva". En Alberto Bernabé, José Antonio Berenguer, Margarita Cantarero y José Carlos de Torres (eds.), Presente y futuro de la Lingüistica en España. La Sociedad de Lingüística 30 años después, págs. 641-648. Madrid, SEL.

Wardaugh, Ronald (1986): An Introduction to Sociolinguistics. Londres, Blackwell. [Traducción a] gallego manejada: Introducción á sociolingüística. Santiago de Compostela, Universidad de Santiago de Compostela.]

Weinreich, Uriel (1953): Languages in Contact. Nueva York, Linguistic Circle of New York.

Woolard, Kathryn A. (1999): "Simultaneity and Bivalence as Strategies in Bilingualism", Journal of Linguistic Anthropology, 8(1), págs. 3-29. 